\title{
OCORRÊNCIA DE Trichogramma pretiosum RILEY (HYMENOPTERA: TRICHOGRAMMATIDAE) EM OVOS DE Bonagota cranaodes (MEYRICK) (LEPIDOPTERA: TORTRICIDAE) EM MACIEIRA ${ }^{1}$
}

\author{
LINO BITTENCOURT MONTEIRO ${ }^{2}$, ALEXANDRE DE SOUZA ${ }^{3}$, EDSON LUIZ BELLI $^{3}$, RANYSE BARBOSA QUERINO \\ DA SILVA ${ }^{4}$, ROBERTO ANTONIO ZUCCHI ${ }^{4}$
}

\begin{abstract}
RESUMO - A lagarta-enroladeira Bonagota cranaodes (Meyrick) é nativa da América do Sul, sendo importante praga em macieira no Brasil. O controle é realizado exclusivamente com inseticidas, e o controle biológico é pouco explorado, motivo pelo qual há necessidade de conhecer os inimigos naturais da lagarta-enroladeira. O objetivo deste trabalho foi verificar a ocorrência de parasitóides de ovos de B. cranaodes. Os levantamentos foram realizados de janeiro de 2000 a novembro de 2001, coletando posturas em folhas de macieiras, Hortência (Hydrangia macrophyla) e Hera (Hedera sp.), em Fraiburgo, Santa Catarina. No período, foram observadas 6,6\% de posturas parasitadas, sendo que, destas, 22,3\% dos ovos estavam parasitados. Os parasitóides foram identificados como Trichogramma pretiosum Riley. Este é o primeiro registro de ocorrência de T. pretiosum parasitando ovos de B. cranaodes em pomares comerciais de macieira no Brasil.
\end{abstract}

Termos para indexação: Controle biológico, parasitóide de ovos, lagarta-enroladeira da macieira

\section{OCCURRENCE OF Trichogramma pretiosum Riley (Hymenoptera: Trichogrammatidae) ON EGGS OF Bonagota cranaodes (Meyrick) (Lepidoptera: Tortricidae) IN APPLE TREE}

ABSTRACT - Apple leaf roller, Bonagota cranaodes (Meyrick) is native from South America and it is one of the most important apple pests in Brazil. Its control is exclusively done by insectides and biological control isn't frequently applied. In this way is necessary to know the parasitoids associated with apple leaf roller. The objective of this study was to detect the parasitism occurrence on B. cranaodes eggs in the State of Santa Catarina (Brazil). From January 2000 to November 2001; 1,700 masses were collected on leaves of apple tree, Hydrangea macrophyla and Hedera sp. in Fraiburgo, Santa Catarina State. During this period egg parasitism was registered in $6.6 \%$ of masses where $22.3 \%$ of the eggs suffered parasitism. The parasite identified was Trichogramma pretiosum. This is the first record of parasitizing of Trichogramma pretiosum in B. cranaodes eggs in commercial apple orchards in Brazil.

Index terms: Biological control, egg parasitoid, apple leaf roller

Bonagota cranaodes (Meyrick) (Lepidoptera: Tortricidae) é uma importante praga na cultura da macieira, causando perdas anuais de $3 \%$ a 5\% da produção (Botton, 1999). Originária da América do Sul, tem sido registrada na Argentina (Meyrick, 1937), Uruguai (Nuñez et al., 1988) e nas mais importantes zonas produtoras de macieira no Brasil (Lorenzato, 1984). Os danos são causados pelas lagartas que raspam a casca das maçãs, provocando a depreciação do fruto. As posturas são realizadas na parte adaxial das folhas em forma de uma massa, contendo, em média, 40 ovos por postura.

O controle de $B$. cranaodes é realizado exclusivamente com inseticidas e não tem proporcionado resultados satisfatórios. $\mathrm{O}$ controle biológico poderia ser uma alternativa ao químico, entretanto poucos são os estudos e registros de inimigos naturais associados à praga. Levantamentos de inimigos naturais foram feitos por Eiras et al. (1992) e Botton (1999), sendo identificados himenópteros que parasitam lagartas; entretanto, parasitóides de ovos da lagarta-enroladeira não foram verificados nestes estudos e por Fonseca (2001).

O presente estudo teve como objetivo verificar a ocorrência de parasitóides de ovos de $B$. cranaodes em pomar comercial de macieira conduzido com controle biológico aplicado de ácaros, em Fraiburgo-SC.

O estudo foi realizado em uma área de 20 ha da empresa Agrícola Fraiburgo S.A, Fraiburgo-SC, de janeiro de 2000 a novembro de 2001. Para verificar a presença de parasitóides de ovos, coletaram-se folhas de macieira durante o período vegetativo e em folhas de Hortência (Hydrangia macrophyla) e Hera (Hedera sp.) durante o inverno, período em que as macieira não apresentam folhas. H. macrophyla) e Hedera sp. desenvolvem-se espontaneamente nas ruas internas e bordaduras da área de estudo. As coletas foram realizadas em $4 \mathrm{~h}$ durante dois dias consecutivos, nas datas de amostragem contidas na Tabela 1. As posturas coletadas foram enviadas para o laboratório de Manejo Integrado de Pragas da Universidade Federal do Paraná, em Curitiba, onde as posturas foram individualizadas em tubos de vidro $(8 \times 2 \mathrm{~cm}) \mathrm{e}$ mantidas em condições controladas $\left(25 \pm 1^{\circ} \mathrm{C}\right.$ e $60 \pm 10 \%$ UR) para o desenvolvimento dos parasitóides.

Foram coletadas 1.700 posturas em 17 levantamentos, sendo que o parasitismo em ovos de B. cranaodes foi observado na maioria das coletas (Tabela 1), exceção feita entre 6 de outubro de 2000 e 15 de janeiro de 2001, quando não foram encontradas posturas no pomar. $\mathrm{O}$ monitoramento realizado com armadilha contendo feromônio, comprovou

TABELA 1 - Parasitismo (\%) de posturas e de ovos de B. cranaodes por T. pretiosum em pomar de macieira, Fraiburgo, SC, 2000/01.

\begin{tabular}{cccccc}
\hline & \multicolumn{3}{c}{ Postura $^{1}$} & & \multicolumn{2}{c}{ Ovos $^{1}$} \\
\cline { 2 - 3 } \cline { 5 - 6 } Período & $\begin{array}{c}\text { Número } \\
\text { total }\end{array}$ & $\begin{array}{c}\text { Parasitismo } \\
(\%)\end{array}$ & & $\begin{array}{c}\text { Número } \\
\text { total }\end{array}$ & $\begin{array}{c}\text { Parasitismo } \\
(\%)\end{array}$ \\
\hline 04-jan-00 & 109 & 9,2 & & 409 & 22,7 \\
10-fev-00 & 89 & 7,9 & & 125 & 16,0 \\
27-abr-00 & 271 & 5,9 & & - & - \\
28-ago-00 & 187 & 1,6 & & 73 & 17,8 \\
06-out-00 & 36 & 0,0 & & - & - \\
05-nov-00 & 0 & 0 & & 0 & 0 \\
11-dez-00 & 0 & 0 & & 0 & 0 \\
15-jan-01 & 0 & 0 & & 0 & 0 \\
22-fev-01 & 74 & 5,4 & & 107 & 12,1 \\
22-mar-01 & 21 & 4,8 & & 23 & 21,7 \\
02-ago-01 & 346 & 1,2 & & 135 & 11,9 \\
22-ago-01 & 314 & 8,6 & & 599 & 21,5 \\
29-set-01 & 148 & 20,9 & & 911 & 28,1 \\
22-out-01 & 14 & 14,3 & & 100 & 20,0 \\
26-out-01 & 13 & 23,1 & & 164 & 16,5 \\
06-nov-01 & 45 & 8,9 & & 314 & 25,8 \\
11-nov-01 & 33 & 3,0 & & 72 & 5,6 \\
\hline
\end{tabular}

${ }^{1}$ Ovos coletados em folhas de macieira, Hedera sp. e Hydrangia macrophyla

\footnotetext{
'(Trabalho 018/2003). Recebido: 16/02/2003. Aceito para publicação: 05/03/2004.

${ }_{2}^{2}$ Depto. de Fitotecnia e Fitossanitarismo, SCA/UFPR, Caixa Postal 19061, 81.531-990, Curitiba-PR. lbmonteiro@terra.com.br,

${ }_{3}^{3}$ Agrícola Fraiburgo Ltda., Rod. Sc. 453, km 35, 89.580-000 Fraiburgo-SC. alex @ agricolafraiburgo.com.br.

${ }^{4}$ Depto. de Entomologia, Fitopatologia e Zoologia Agrícola, ESALQ/USP, Caixa Postal 9, 13418-900, Piracicaba-SP. razucchi@esalq.usp.br, ranyse@ @inpa.gov.br.
} 
a ausência de adultos de $B$. cranaodes neste período.

Verificou-se que, em média, foram parasitadas $6,6 \%$ das posturas e que, em cada postura, houve, em média, $22,3 \%$ de ovos parasitados. Os maiores índices de parasitismo ocorreram em setembro e outubro de 2001.

Os parasitóides de ovos foram identificados como Trichogramma pretiosum Riley (Hymenoptera: Trichogrammatidae) e estão depositados na coleção do Departamento de Entomologia, Fitopatologia e Zoologia Agrícola da ESALQ/USP. No Brasil, este é o primeiro registro da ocorrência de parasitismo de ovos de B. cranaodes por T. pretiosum em pomares de macieira comercial.

\section{AGRADECIMENTOS}

Os autores agradecem à Agrícola Fraiburgo S. A., pelo apoio financeiro.

\section{REFERÊNCIAS BIBLIOGRÁFICAS}

BOTTON, M. Bioecologia e controle de Bonagota cranaodes (Meyrick, 1937) (Lepidoptera: Tortricidae) na cultura da macieira. 1999, 73p.

Tese (Doutorado em Entomologia) Escola Superior de Agricultura “Luiz de Queiroz”, Universidade de São Paulo, Piracicaba, 1999.
EIRAS, A. E.; A. KOVASLESKI, E. F.; VILELA, C. E. P.; SOUZA, R. S. F.; FRIGHETO, N. FRIGUETTO; L. R. K. DELMORE. Observações preliminares sobre a ocorrência do parasitismo natural na lagartaenroladeira Phtheochroa cranaodes (Meyrick) (Lepidoptera: Tortricidae) em pomares de macieira em Vacaria (RS). In: SIMPÓSIO DE CONTROLE BIOLÓGICO, 3., 1992, Águas de Lindóia-SP. Resumos... Jaguariúna: CNPDA/EMBRAPA, 1992.p. 304.

FONSECA, F. L. Ocorrência de Trichogramma (Hymenoptera: Trichogrammatidae) nas localidades de Pelotas, Bento Gonçalves e Vacaria-RS e aspectos bioecológicos em ovos de Bonagota cranaodes (Meyrick, 1937) (Lepidoptera: Tortricidae). 2001. 59f. Tese (Doutorado em Fitossanidade) Universidade Federal de Pelotas, Pelotas, 2001

LORENZATO, D. Ensaio laboratorial de controle da "traça-da-maçã Phtheochroa cranaodes Meyrick, 1937 com Bacillus thuringiensis Berliner e inseticidas químicos. Agronomia Sulriograndense, Porto Alegre, v. 20, p. 157-163, 1984.

MEYRICK, E. Phtheochroa cranaodes. Exotic Microlepidoptera, V.5 p. 149-150, 1937.

NUNEZ, S.; GARCIA, S.; PAGANI, C.; MAESO, D. Guia para el manejo integrado de plagas y enfermidades en frutales. Las Brujas: INIA. 1998, 117p. (Boletin de Divulgacion, 66). 